\title{
EL YACIMIENTO DE PLEISTOCENO SUPERIOR DE CUEVA BAJONDILLO (TORREMOLINOS, MÁLAGA): PROCESOS DE INVESTIGACIÓN Y DE DIFUSIÓN
}

\section{THE UPPER PLEISTOCENE SITE OF CUEVA BAJONDILLO (TORREMOLINOS, MÁLAGA, SPAIN). RESEARCH AND DISSEMINATION}

\author{
por \\ ANA BALDOMERO NAVARRO* \\ JOSÉ E. FERRER PALMA* \\ IGNACIO MARQUÉS MERELO*
}

RESUMEN Se presenta una síntesis de los diversos trabajos de investigación realizados en la Cueva Bajondillo y de las publicaciones presentadas derivadas de los mismos desde 1989 hasta la actualidad, centrándose en el tramo estratigráfico correspondiente al Pleistoceno Superior

\begin{abstract}
It is the aim of this article to present a synthesis of the state of research carried out at Cueva Bajondillo and the publications dealing with this archaelogical site written from 1989 to the present moment specially focusing on the stratigraphic plot corresponding to the Upper Pleistocene.
\end{abstract}

Palabras claves Asentamiento en cueva. Cueva Bajondillo. Industrias líticas del Pleistoceno Superior. Transición Paleolítico Medio-Paleolítico Superior.

Key words Cave settlement. Cueva Bajondillo. Upper Pleistocene lithic industries. Transition Middle Paleolithic-Upper Paleolithic.

Desde que en 1990 dábamos a conocer el yacimiento arqueológico de la Cueva Bajondillo (Baldomero Navarro, Marqués Merelo y Ferrer Palma 1989-90), se han venido publicando diversos trabajos sobre el mismo en distintas revistas y actas de reuniones científicas cuyos temas guardaban alguna relación con el marco arqueológico o geológico del yacimiento y en los que se han ido presentando las novedades que

* Área de Prehistoria de la Universidad de Málaga. 
el estudio continuado de la documentación obtenida en los trabajos de excavación iba aportando, siempre a nivel de conclusiones iniciales y por lo tanto pendientes de confirmar una vez concluido todo el estudio analítico, que se ha visto ampliado con la actuación llevada a cabo en el yacimiento en el verano de 2000.

Transcurrida algo más de una década desde esa primera noticia, a la que han seguido varias publicaciones, creemos llegado el momento de presentar una síntesis de lo hecho y publicado hasta ahora en y sobre el yacimiento, recogiendo los distintos aspectos tratados e incorporando los objetivos de la actuación de 2000; esta recapitulación servirá al propio tiempo como punto de partida para próximos trabajos, en los que expondremos los resultados obtenidos, en las distintas vertientes, a partir de esa reciente actuación, de la que todavía estamos recibiendo datos.

Como creemos que es ya sabido, el yacimiento se encuentra situado en la zona de Bajondillo, sobre las playas del mismo nombre, en el término municipal de Torremolinos, constituyendo los sedimentos arqueológicos el relleno de una cueva formada en un travertino que, además de las alteraciones debidas a procesos naturales, se ha visto sometida a una larga actuación antrópica no excesivamente bien conocida, que la ha cortado verticalmente y que ha culminado, a partir de la década de 1960-1970, con la total urbanización del enclave (Marqués Merelo y Ferrer Palma 1991: 15; fig. 1).

El descubrimiento del yacimiento se produce en 1989, precisamente con motivo de las obras de construcción del complejo de apartamentos Torresol, llevándose a cabo todas las actuaciones arqueológicas, siempre con carácter de urgencia por encontrarse el yacimiento en un solar en proceso de construcción de inmuebles, desde ese momento y a lo largo del verano de ese mismo año.

Dado el interés que, incluso desde una visión muy general, presentaba la secuencia cultural del yacimiento por su amplitud cronológica, abarcando diversos contextos que arrancaban del Paleolítico Medio y que culminaban en momentos de Prehistoria Reciente, nuestras primeras publicaciones tienen lugar poco tiempo después de las actuaciones arqueológicas, concretamente en 1990 (Baldomero Navarro, Marqués Merelo y Ferrer Palma 1989-90) y 1991 (Baldomero Navarro, Ferrer Palma y Marqués Merelo 1991; Marqués Merelo y Ferrer Palma 1991).

Contribuía también a la realización de estas primeras publicaciones el hecho de que, poco después de concluidas las excavaciones, en 1990, el tribunal que juzga la Tesis Doctoral de J.L. Sanchidrián (Sanchidrián Torti 1990) reúne en Málaga a los Drs. Fortea Pérez, Moure Romanillo y Villaverde Bonilla. Estos tienen la oportunidad de ser los primeros en analizar algunos materiales obtenidos en la recién terminada excavación de Bajondillo, aún inéditos, y se muestran de acuerdo con los datos preliminares, instándonos a dar a conocer lo más rápidamente posible las evidencias que hasta aquel momento resultaban más claras.

En estos breves artículos señalábamos sobre todo las circunstancias en las que se había producido el descubrimiento del yacimiento, la situación en la que se encontraba el lugar en el que se ubica, las actuaciones arqueológicas de urgencia que habíamos llevado a cabo y las dificultades de diversa índole en las que debieron realizarse aquellas y que las condicionaron en gran medida. Respecto al espacio que contenía los sedimentos excavados, los primeros resultados del estudio geológico indicaban que se trataba de una cueva que se había formado en materiales travertínicos que corresponden a depósitos post-orogénicos originados entre las unidades de Blanca y el Maláguide, que pertenecen al Bético Inferior; estas referencias de carácter geológico se veían complementadas con la incorporación de las fechaciones absolutas obtenidas por el equipo de J. J. Durán para el travertino de Torremolinos por el método Electron Spin Resonance (E.S.R.) y el de Uranio/Torio, que situaban en aquel momento la formación del travertino entre 23.300 y 27.300 B.P. (Durán, Grün y Soria 1988).

Puesto que el estudio analítico del material recuperado apenas si se había iniciado por la cercanía a las fechas de la excavación arqueológica, dentro del conjunto de la secuencia cultural en estas primeras publicaciones nos referimos únicamente a los contextos de más clara clasificación por la expresividad de la mayoría de los tipos líticos que habíamos podido identificar y correlacionar estratigráficamente en el 
transcurso de la excavación, concretamente al Musteriense, al Solutreogravetiense y a momentos neolíticos que llegaban a conectar con los inicios de la Edad del Cobre, dando tan sólo datos técnicos y tipológicos muy generales de los mismos por las razones ya expuestas, sin desarrollar dichas analíticas ni incorporar estudios estadísticos. El hecho de que los sedimentos iniciales de la secuencia obtenida pertenecieran al Musteriense nos llevaba a considerar como recientes las fechaciones absolutas conocidas para el travertino y que anteriormente señalamos.

En resumen, con estas primeras y breves publicaciones pretendíamos dar a conocer el yacimiento por la importancia que pensábamos revestía a partir de los primeros contextos culturales identificados y que hemos mencionado anteriormente, y en las que, en relación a la secuencia cultural, únicamente nos referíamos, y a muy grandes rasgos, a los conjuntos industriales que habíamos podido diferenciar fácilmente por presentar unas características muy definidas, y en términos similares a la fase de Prehistoria Reciente, insistiendo en todo momento en el carácter general y provisional de esas primeras conclusiones, dada la proximidad temporal existente entre los trabajos de excavación y estas publicaciones.

Pero en aquella visita a Málaga, los Drs. Fortea Pérez, Moure Romanillo y Villaverde Bonilla nos animaban a estudiar a fondo los resultados globales de la excavación, aconsejándonos apoyarlos con el estudio de las analíticas obtenidas a través de la actuación de urgencia y a ser posible con dataciones absolutas de la columna estratigráfica.

En este último sentido, a través del proceso de excavación se habían obtenido algunas series de muestreos, encaminadas esencialmente a la realización de analíticas sedimentológicas y polínicas, dejándose para más tarde el intento de ampliar las tomas de muestras para dataciones, toda vez que las obtenidas con este fin durante el proceso de excavación eran parciales a la columna, escasas y de las que, debido al contexto en el que nos movíamos (la excavación se llevó a cabo a la vez que la construcción de las edificaciones), se podía sospechar que su extracción en algún caso no contaba con las suficientes garantías de aislamiento.

Animados a profundizar en el estudio del yacimiento y cubierto el trámite que, al menos en nuestra Comunidad, obliga a dar a conocer de forma inmediata los resultados preliminares de cualquier excavación de urgencia, pusimos en marcha un proyecto de investigación que, por una parte, estudiara minuciosamente los aspectos técnicos y tipológicos, tan sólo esbozados y referidos muy parcialmente a la secuencia obtenida $\mathrm{y}$, por otra, los dotara de un entramado sólido sustentado por cronologías precisas y datos sobre el medioambiente y sobre las actividades antrópicas llevadas a cabo en él y, finalmente, localizara las fuentes de suministro utilizadas y relacionara el entorno con la utilización del enclave que habíamos documentado.

Para algunos de estos aspectos, en concreto los que precisaban de algún tipo de actuación (comprobaciones estratigráficas, nuevas tomas de muestras... ) contábamos a priori con la posibilidad de acceder a los restos conservados del yacimiento, para lo que se había solicitado a la empresa constructora la reserva de un pequeño espacio al que no se podría acceder desde las edificaciones previstas, tan sólo desde un paso municipal vigilado, y que actuaría como área de conservación donde pudiera controlarse, entre otros factores, la posible vegetación espontánea que presumiblemente iba a producirse sobre el perfil extraplomo resultante. En aquel momento contábamos con que pudiera utilizarse en función de las necesidades de investigación.

Ultimado este proyecto en 1993, incorporamos al mismo a los dos Licenciados de la Universidad de Málaga, Miguel Cortés Sánchez y María Dolores Simón Vallejo, que venían colaborando habitualmente con nuestro Departamento y habían mostrado su interés por los estudios paleolíticos, incorporando el yacimiento de Bajondillo a sus trabajos de Doctorado. El volumen de datos que poseíamos y las perspectivas que se entreveían en aquel momento permitían pensar en la viabilidad de soportar, al menos parcialmente, sus doctorados. Los primeros trabajos llevados a cabo consistieron en sentar la relación entre las nutridas series de artefactos recuperados en el transcurso de la excavación y la columna estratigráfica, habida cuenta que en el sistema de obtención de datos habíamos utilizado, si bien tan sólo en algunos de los sectores de la excavación que en su mayoría permitían por su estrechez referencias seguras, la metodología de excavación 
por capas artificiales, con ubicación tridimensional, que obligaba a reconstruir su posición exacta mediante trabajos de laboratorio.

Esta primera aproximación se limitaba inicialmente a reubicar con exactitud los materiales en los estratos y subestratos identificados en el transcurso de la excavación hasta un total de diecisiete. Esta labor que se desarrolla hasta 1995, tan sólo permitió esbozar un análisis cualitativo de los elementos tipológicos que resultaban más destacados, quedando pendiente de abordar en una segunda fase el estudio global de las industrias con aspectos cuantitativos, tecnológicos y morfológicos. Pero por primera vez se presentaba un cuadro, aunque todavía sólo aproximado, de la secuencia cultural completa del yacimiento, donde estratos que no habían podido ser identificados de forma exacta en el proceso de los trabajos de campo, adquirían ahora una dimensión real lejos de la ambigüedad inicial.

En aquel momento vimos la necesidad de apoyar con dataciones fiables períodos de la secuencia que no parecían ser característicos del ámbito meridional, concretamente los referentes al Paleolítico Superior Inicial, cuya presencia en Bajondillo podía levantar suspicacias, por lo que dimos prioridad a otra de las fases del Proyecto que pasaba forzosamente por una nueva toma de contacto con el yacimiento. En realidad, habíamos intentado este contacto con anterioridad con resultado negativo. Las edificaciones que en su día originaron las actuaciones de urgencia habían producido una serie de conflictos económicos entre mancomunidad de propietarios y empresa constructora que habían desembocado en una orden de embargo preventivo de todo el solar y existía prohibición expresa de acceder al espacio construido y, por lo tanto, imposibilidad legal de visitar el yacimiento.

Nuestras primeras actuaciones en esta dirección nos inclinaron a pensar que la solución del conflicto no se resolvería a corto plazo puesto que en él intervenían los intereses de los propietarios de los inmuebles, aún no recepcionados, los de la empresa constructora, los de la administración local que desde nuestra excavación había variado de pertenecer a Málaga capital para corresponder a Torremolinos, término municipal creado en ese tiempo intermedio, así como los de la administración regional por estar afectado desde el punto de vista arqueológico. Por ese motivo y comprendiendo que estas nuevas conclusiones, a pesar de su provisionalidad, eran merecedoras de un conocimiento público por parte de los investigadores interesados en el tema, becamos a uno de nuestros investigadores, Miguel Cortés Sánchez, en aquel momento miembro del Grupo de Investigación del Área de Prehistoria de la Universidad de Málaga, para que acudiera al Coloquio Internacional "El món mediterrani després del Peniglacial (18.000-12.000 B.P)" a celebrar en Bañolas (Gerona) en mayo de 1995 y, juntamente con María Dolores Simón Vallejo, miembro también por aquellas fechas del mismo grupo de investigación, para que dieran a conocer los avances conseguidos y las aportaciones al Paleolítico de Andalucía que suponían.

Allí es, por lo tanto, cuando comienzan a aparecer los nuevos datos que estábamos en disposición de ofrecer y que venían a modificar la presentación preliminar de los años 1990 y 1991 (Cortés Sánchez y Simón Vallejo 1997). El interés que suscitan y el conocimiento de las dificultades que soportaba el yacimiento para cotejar datos se traduce en una intervención directa del Presidente de la U.I.S.P.P., Dr. D. Dominique Sacchi, que se refleja en una instancia a la Presidencia de la Junta de Andalucía donde se manifiesta dicho interés y se solicita el apoyo e intervención de la administración andaluza.

Desde la presentación de aquellos nuevos datos que habían resultado del estudio clasificatorio inicial, hemos venido permitiendo la publicación de algunos aspectos destacados que la elaboración de la primera de las tesis doctorales, basada parcialmente en la Cueva Bajondillo, iba revelando. Así, en abril-mayo de 1996, se presentan al I Simposio de Prehistoria Cueva de Nerja "Las Culturas del Pleistoceno Superior en Andalucía" Homenaje al profesor Dr. D. Francisco Jordá Cerdá (Cortés Sánchez y Simón Vallejo 1998), donde además se da la oportunidad a todos los investigadores interesados en contemplar una selección de piezas de todos los estratos del yacimiento (Sanchidrián Torti 1998); en agosto de 1998 se explican mediante un póster en el Congreso Internacional "Gibraltar and the Neanderthals, 1948-1998" (Cortés Sánchez 
1998) que es acompañado por la consiguiente publicación (Cortés Sánchez 2000); por último, en octubre de 1998 se exponen en el Congreso "Les premiers hommes modernes de la Péninsule Ibérique" celebrado en Vila Nova de Fozcôa (Guarda, Beira, Portugal) (Cortés Sánchez y Simón Vallejo 2001).

También han sido usados los resultados que iban conociéndose de la investigación de la Cueva Bajondillo en monografías, colaboraciones o artículos no específicos de este yacimiento y, mayoritariamente, de autoría compartida por nuestros doctorandos (Cortés Sánchez, Muñoz Vivas, Sanchidrián Torti y Simón Vallejo 1996; Cortés Sánchez y Sanchidrián Torti 1997; Cortés Sánchez y Sanchidrián Torti 1998; Cortés Sánchez y Simón Vallejo 2000).

A través de estos avances hemos ido dibujando el perfil actual del yacimiento, necesariamente aún provisional. Así, estamos en disposición de situar a Cueva Bajondillo en los escarpes inmediatos a la llanura litoral, en el conocido como Tajo de Torremolinos, abierta al farallón travertínico que se dispone sobre la Playa del Bajondillo y que ahora se nos presenta considerablemente envejecido (Cortés Sánchez y Simón Vallejo 2000) con respecto a las fechas (Durán, Grün y Soria 1988) que habían proporcionado analíticas anteriores tratadas mediante E.S.R (25.300-26.500 $\pm 15 / 20 \%$ B.P.) y U. $(27.300 \pm 1.700$ B.P.). Con una serie estratigráfica compuesta inicialmente por 17 niveles (Cortés Sánchez y Simón Vallejo 1997: 278) de cuyo intervalo paleolítico se han podido relacionar, por la expresividad de sus artefactos, 12 hasta la fecha, que, de techo a muro, afectan a los niveles 6 a 17. Los niveles superiores que pudieron ser excavados con garantías (la presencia de algunos de ellos, parcial a la estratigrafía del yacimiento, sólo pudo documentarse muy escasamente o incluso sólo de forma gráfica, puesto que habían sido o muy alterados o eliminados previamente por el acondicionamiento del solar en la fase previa a las edificaciones), pertenecen al tramo de Prehistoria Reciente y su estudio aún no ha sido abordado en profundidad.

Los niveles 6,7 y 8 se corresponden con la fase solutreogravetiense que dimos a conocer a través de los primeros avances (Marqués Merelo y Ferrer Palma 1991). En ellos predominan los buriles, preferentemente sobre truncadura aunque no sean infrecuentes los de ángulo y diedros, sobre los raspadores, bien simples o sobre lámina retocada y algunos cortos y espesos; entre los dorsos destacan las puntas escotadas, las escotaduras distales y algunas microgravettes y laminitas de dorso (fig. 3, B). Este conjunto nos inclinó a considerar un momento avanzado de la fase propuesta.

Para el nivel 9, tras el estudio de todos los artefactos que presumiblemente pueden relacionársele a pesar de las dificultades que presentó su documentación en el transcurso de la excavación de 1989, se ha aventurado su relación en una fase plena del Solutrense, aunque no pueda descartarse un momento algo más avanzado, En él no aparecen los elementos escotados anteriores y los dorsos disminuyen sensiblemente; entre los materiales que podrían llegar a adscribírsele, se documentan pequeños fragmentos de puntas de cara plana así como algunos fragmentos de piezas en las que el retoque plano es evidente y que han sido catalogadas como probables hojas de laurel. El resto del material se concreta en un porcentaje mayoritario de buriles, predominando los realizados sobre escotaduras, frente a los raspadores, en la misma tendencia de los niveles anteriores (fig. 3, A)

Bajo este nivel, el 10, no ha proporcionado, tras un estudio detenido, ningún indicio de retoques planos y sí en cambio destaca en él la presencia de retoques abruptos, presentes en un extremo distal de punta de La Gravette y en varias microgravettes, puntas de dorso curvo y laminitas de dorso, permitiendo la atribución al Gravetiense que, por la tipología de sus puntas, quizás pudiera concretarse en una fase relativamente antigua (Cortés Sánchez y Simón Vallejo 1997: 282). Completan el cuadro material algunas truncaduras, así como buriles, de nuevo en mayor cantidad que los raspadores, en especial diedros y sobre truncadura (fig. 2, B), integrando todo ello un conjunto en realidad exiguo, resultado de una escasa y a la vez diversificada frecuentación antrópica de la cueva debida, probablemente, al peligro que debió suponer la caída de fragmentos de visera que aparecen documentados entre los sedimentos. 
El nivel 11 posee unas características bien distintas a las del nivel superior expuesto anteriormente. En el apartado tecnológico habría que señalar la disminución sensible del modo abrupto frente a un claro dominio del simple, juntamente con el mantenimiento de núcleos destinados a la fabricación de hojas y hojitas, a nivel tecnológico. A nivel tipológico, destaca la inversión de la proporción mantenida en los niveles superiores a favor ahora de los raspadores, especialmente en hocico pero también carenados, frente a los buriles, generalmente diedros; además, la presencia de algunas laminitas con retoque marginal simple, entre las que se hubiera podido admitir algún subtipo Dufour si no fuera por la imposibilidad absoluta de correlacionarlos con el nivel, al haber sido localizadas en áreas del yacimiento fuertemente alteradas. Por último, se localizan así mismo muescas y denticulados (fig. 2, A). Esta variación sustancial de tendencias tecnológicas, con respecto a los niveles inferiores que veremos a continuación, y tipológicas, en relación a los superiores ya vistos, ha motivado la clasificación de Auriñaciense para el nivel 11. Como puede desprenderse de lo dicho, resulta una clasificación más aproximada de lo que desearíamos, por lo que hasta la fecha tan sólo se ha propuesto de forma genérica como Auriñaciense sensu lato (Cortés Sánchez y Simón Vallejo 1997: 283 ), ya que carecemos de caracterizadores más concretos procedentes de la industria ósea, ausente por el momento en la secuencia.

La adscripción de los niveles 12 y 13, debido a sus carencias documentales (escaso espesor de sus sedimentos, parcialidad en el área investigada, dificultad de identificación cromática, posición acuñada, parquedad de tipos de útiles, reducido número de elementos artefactuales en general, ambigüedad de caracteres técnicos...), ha sufrido en el transcurso de la investigación algunas variaciones interpretativas a medida que se iban conociendo más datos sobre ellos. Así, en un primer momento, se consideraron en conjunto debido a que su presencia sólo pudo ser documentada convenientemente en las inmediaciones del travertino, en su sector centro-sur, donde se interestratificaban dificultando su aislamiento en el proceso de la excavación, suponiéndoles integrantes de las series Paleolítico Medio por la presencia de componentes industriales de afinidad musteriense, juntamente con el resto de los niveles inferiores, hasta el 17 (Cortés Sánchez y Simón Vallejo 1997: 284; Cortés Sánchez y Simón Vallejo 1998: 40), e inclinando a considerar la existencia de una ruptura clara entre ellos y el nivel 11 superpuesto (Cortés Sánchez y Simón Vallejo 1997: 284; Cortés Sánchez y Simón Vallejo 1998: 42).

Posteriormente, se ha barajado la posibilidad de que el nivel 12 formara parte de la serie Paleolítico Superior, como un "área particular" del nivel 11 (Cortés Sánchez 1998; Cortés Sánchez y Simón Vallejo 2001: 108). Su presencia, como ya se ha dicho, sólo parcial a la secuencia, hacía pensar más en una variante sedimentológica que en un nivel con entidad propia y, en esta línea, la apariencia de algunos indicadores tecnológicos, a pesar de no haber sido aislados convenientemente en el transcurso de la excavación por las dificultades ya expresadas, algún núcleo así como láminas y laminitas, podía permitir hipotetizar sobre su dependencia del ya citado nivel 11 (Cortés Sánchez 2000: 125). De acuerdo a esto, la hipótesis más lógica para el nivel 13 era considerarlo el epílogo del musteriense que se localizaba en el paquete sedimentario inferior (Cortés Sánchez 1998).

No obstante, la posición en la estratigrafía de estos niveles hacía ver el peligro de una definición simple, en la que se le atribuyera al 13, sin más, la presencia de los otros componentes de afinidad musteriense, creando un binomio nivel $12=$ Auriñaciense $/$ nivel $13=$ Musteriense, lo que precisa de otros apoyos, bien tecnotipológicos, bien sedimentológicos y cronológicos; por ello se plantea postponer su adscripción definitiva (Cortés Sánchez 2000: 125; Cortés Sánchez y Simón Vallejo 2001: 108) para más adelante, obviamente hasta no contar con dichos apoyos.

Desde los primeros avances, hemos venido hablando en conjunto del tramo sedimentario correspondiente al Paleolítico Medio con presencia de claros tecnocomplejos musterienses (Baldomero Navarro, Marqués Merelo y Ferrer Palma 1989-90; Baldomero Navarro, Ferrer Palma y Marqués Merelo 1991) que se concretaron en la secuencia, como acabamos de exponer, de los niveles 12 a 17 (Cortés Sánchez y Simón 
Vallejo 1997: 284; Cortés Sánchez y Simón Vallejo 1998: 40), limitándose posteriormente con claridad a los niveles 14 a 17 (Cortés Sánchez 2000; Cortés Sánchez y Simón Vallejo 2001). De estos últimos, tan sólo se han publicado datos concretos acerca del nivel 14 (Cortés Sánchez 1998; Cortés Sánchez 2000; Cortés Sánchez y Simón Vallejo 2001), manteniendo los restantes inéditos, al reservar los resultados de su estudio hasta el momento de la defensa (inminente en el momento de la entrega de este trabajo) de la tesis doctoral de Cortés Sánchez.

Por ello, siguiendo la línea de síntesis que nos hemos propuesto en este trabajo, sólo haremos mención de los datos difundidos sobre el nivel 14 que responde, con claridad, al último nivel del tramo sedimentario del Paleolítico Medio general al área investigada. Ha quedado definido este nivel como propio de un Musteriense de facies rica en denticulados por el alto índice del grupo, Im-d alrededor del $40 \%$, presencia de cuchillos de dorso natural, con una pobre representación de las raederas, IR $<15 \%$, Grupo III alrededor del 10\%, en el que los buriles y perforadores mantienen una proporción superior a los raspadores (IB >IG; IP>IG) (Cortés Sánchez y Simón Vallejo 2001: 103-104 ). Así mismo, podría citarse la presencia de varias puntas de Tayac y la ausencia de puntas musterienses (Cortés Sánchez1998), que aparecen claramente representadas en la secuencia inferior, niveles 15 a 17 (Baldomero Navarro, Marqués Merelo y Ferrer Palma 1989-90; Baldomero Navarro, Ferrer Palma y Marqués Merelo 1991) (fig. 1). Por último, si bien en la gestión de los recursos abióticos, sílex mayoritariamente $(90 \%)$ frente a cuarcitas y calizas $(10 \%)$, aparece frecuentemente el método Levallois junto a los núcleos de gestión discoidal (Cortés Sánchez 2000: 125), los soportes levallois son escasamente utilizados para su transformación (Cortés Sánchez1998).

Como es obvio, después de esta síntesis, todas las aportaciones que con carácter de avances se han venido produciendo, se han ceñido a aclarar la secuencia estratigráfica por medio de las novedades que el desarrollo del estudio, básicamente tecnomorfológico y tipológico de las colecciones líticas recuperadas, iba permitiendo. Es cierto que no hemos presentado hasta ahora, más que de forma incidental (Cortés Sánchez 2000: 131), aspectos que se podrían echar de menos, como la economía desarrollada por los que utilizaron Cueva Bajondillo, su posible organización, la tipología del enclave....Algunos de ellos se expondrán en la ya citada tesis doctoral de Cortés Sánchez, otros, por el momento, son muy difíciles de concretar debido a las carencias específicas del yacimiento. En este sentido, se ha destacado la escasísima colección faunística rescatada en el transcurso de la excavación (Cortés Sánchez 2000: 131) que es el resultado de una carencia real de restos, constatada así mismo en la última actuación de documentación realizada en agosto de 2000 , carencia con la que podría relacionarse el mal estado que presenta el colágeno de los escasos restos óseos conservados, pendiente de explicación por medio de las analíticas. Por otra parte, la colección malacológica es algo superior, aunque no mucho más y tampoco permite por el momento inferencias especiales de tipo dietético.

Las reacciones que ha provocado la presencia de un Paleolítico Superior Inicial, con conjuntos industriales que consideramos como Auriñaciense s. l. y Gravetiense, en la Cueva Bajondillo, han sido muy contrapuestas por las implicaciones que ello representaba en la problemática sobre la transición Paleolítico Medio/Paleolítico Superior en Andalucía, con una muy escasa documentación referida a esos conjuntos industriales de Paleolítico Superior Inicial (Sanchidrián Torti; Simón Vallejo; Cortés Sánchez y Muñoz Vivas 1996: 51-55).

De esta forma nos encontramos con posturas contrarias o que manifiestan grandes dudas respecto a la consideración de Auriñaciense y Gravetiense en Bajondillo, que se plantean con argumentos poco consistentes en nuestra opinión (Castañeda Fernández 1997-98: 8-10; Castañeda Fernández y Herrero Lapaz 1999: 130-133), sin tener en cuenta, quizás debido a una lectura apresurada, el carácter general y provisional de las conclusiones que aportábamos en las primeras publicaciones (Paniagua Pérez 1998: 56-57) y que cuando nos referimos en ellas al Solutreogravetiense señalamos que corresponde a "Los niveles sedimentológicos que hemos investigado hasta la fecha.....", utilizando el término investigación en el sentido de la contrastación en laboratorio de los trabajos de campo (Marqués Merelo y Ferrer Palma 199: 141), desconociendo las publicaciones de 1997 y guiándose únicamente por las exposiciones orales (Paniagua Pérez 
1998: 63-67) y cayendo incluso en manifestaciones desafortunadas (Muñiz Pérez y Paniagua Pérez 1996), cuando no se conoce directamente ni a los investigadores del yacimiento, ni las actuaciones arqueológicas realizadas en el mismo ni el material lítico que nos servía de base para hablar de un Paleolítico Superior Inicial en Bajondillo.

Muy distinta es la opinión manifestada por varios investigadores, cuyo profundo conocimiento del tema que nos ocupa es indiscutible. En este sentido debemos mencionar por una parte a J. Zillhao, quien recoge el Auriñaciense de Bajondillo en un mapa de dispersión de yacimientos paleolíticos peninsulares (Zillhao 2000: fig. 12-1) y por otra a J. L. Sanchidrían (Sanchidrián Torti, Simón Vallejo, Cortés Sánchez y Muñoz Vivas 1996: 52; Sanchidrián Torti 2000: 546) y G. Vega (Alcolea 1997: 416), quienes conociendo personalmente desde tiempo atrás a los investigadores del yacimiento y después de analizar directamente los conjuntos industriales a los que nos estamos refiriendo, no ponen objeción alguna al hablar de Auriñaciense y de Gravetiense en la Cueva Bajondillo, calificando el segundo de ellos a ambos conjuntos industriales como claros (Alcolea 1997: 416).

De todas formas y puesto que los avances que se fueron publicando a partir de 1996 se apoyaban exclusivamente en análisis de conjuntos industriales, en el verano de 2000 , una vez subsanados los problemas de intervención a finales de 1999, llevamos a cabo una actuación en el yacimiento de cara a aumentar la documentación de que disponíamos para corroborar dichos avances y ampliar en general el conocimiento del yacimiento.

Seis años pues, entre 1993 y 1999, ha costado superar las dificultades legales que impedían nuestro acceso al yacimiento y por lo tanto obtener los muestreos que complementasen y en su caso sustituyesen a los ya obtenidos durante la campaña de excavaciones de 1989. Subsanadas estas dificultades y contando con la inestimable ayuda del Patronato de la Cueva de Nerja y del Excelentísimo Ayuntamiento de Torremolinos, se pudo volver a intervenir sobre los sedimentos conservados obteniendo nuevas series de muestreos. Esta actuación ha estado encaminada básicamente a la toma de muestras para diversas analíticas, como por ejemplo la palinológica, realizada por P. López, la sedimentológica y microestratigráfica, llevada a cabo por M. Bergadá, la geológica, que desarrollan F. Carrasco, B. Andreo y J. J. Durán, y, por supuesto, las relativas a fechaciones absolutas, que han incluido los sistemas de $\mathrm{C}-14$ y Termoluminiscencia para los sedimentos arqueológicos y Uranio/Torio para el travertino.

El estudio de toda esta analítica no ha concluido aún, aunque estamos en condiciones de avanzar que las fechaciones absolutas obtenidas avalan las consideraciones que hemos venido defendiendo respecto al Paleolítico Superior Inicial de Bajondillo y han envejecido considerablemente, como hemos dicho, el travertino, llevándolo a una cronología totalmente compatible con el Musteriense que ocupa el tramo estratigráfico inferior de la secuencia de Bajondillo. Todos estos resultados han sido expuestos recientemente por el equipo del proyecto en agosto de 2001, sin que hayan sido mínimamente cuestionados en ningún momento, en el Congreso sobre Neanderthals and Modern Humans in Late Pleistocene Eurasia celebrado en el pasado mes de agosto en Gibraltar, en cuyas actas serán publicados (Baldomero Navarro; Cortés Sánchez; Ferrer Palma; Marqués Merelo y Simón Vallejo, en prensa). 


\section{BIBLIOGRAFÍA}

ALCOLEA, J. J. (1997): “Transcripción debates de la Sección”, en BALBÍN BERHMANN, R. de y BUENO RAMÍREZ, P. (Eds) (1997): II Congreso de Arqueología Peninsular. T. I Paleolítico y Epipaleolítico: 415-421. Zamora.

BALDOMERO NAVARRO, A.; MARQUÉS MERELO, I. y FERRER PALMA, J. E. (1989-90): "Intervención de urgencia en el Bajondillo (Torremolinos, Málaga)", Mainake XI-XII: 11-20. Málaga

BALDOMERO NAVARRO, A.; FERRER PALMA, J. E. y MARQUÉS MERELO, I. (1991): "Excavaciones de urgencia en el Bajondillo (Torremolinos, Málaga)", Anuario Arqueológico de Andalucia/1989, III Actividades de Urgencia: 357-359. Sevilla.

BALDOMERONAVARRO, A.; CORTÉS SÁNCHEZ, M.; FERRER PALMA, J.E.; MARQUÉS MERELO, I. y SIMÓN VALLEJO, Ma D. (en prensa): “Cueva Bajondillo (Torremolinos, Málaga, Andalucía). El tránsito Paleolítico Medio-Superior en la mitad meridional de la Península Ibérica”, Nenderthals and Modern Humans in Late Pleistocene Eurasia, Gibraltar.

CASTAÑEDA FERNÁNDEZ, V. (1997-98): "La llegada del Homo Sapiens al Sur de la península Ibérica. Una revisión crítica sobre su estudio", Mainake, XIX-XX: 5-16. Málaga.

CASTAÑEDA FERNÁNDEZ, V. y HERRERO LAPAZ, N. (1999): “La perduración y sustitución de los neandertales en el centro y sur de la península Ibérica. Una revisión crítica para su estudio", Revista Atlántica-Mediterránea de Prehistoria y Arqueología Social 2: 123-150. Cádiz.

CORTÉS SÁNCHEZ, M.; MUÑOZ VIVAS, V. E.; SANCHIDRIÁN TORTI, J. L. y SIMÓN VALLEJO, M. D. (1996): El Paleolítico en Andalucía. Córdoba.

CORTÉS SÁNCHEZ, M. y SIMÓN VALLEJO, Mª D. (1997): “Cueva Bajondillo(Torremolinos, Málaga). Aportaciones al Paleolítico en Andalucía", en FULLOLA, J. M. y SOLER, N. (eds.): El món mediterrani després del Peniglacial (18.000-12.000 B.P), Serie Monográfica, 17, Museu d'Arqueologia de CatalunyaGirona: 275-289. Girona.

CORTÉS SÁNCHEZ, M. y SANCHIDRIÁN TORTI, J. L. (1997): "Dinámica Cultural del Pleistoceno Superior en la costa de Málaga”, en RODRÍGUEZ, J. (Ed.): Cuaternario Ibérico: 334-338. Huelva.

CORTÉS SÁNCHEZ, M. (1998): El final del Paleolítico Medio en la Bahía de Málaga: Cueva Bajondillo (Torremolinos, Málaga, Andalucía). Gibraltar.

CORTÉS SÁNCHEZ, M. y SANCHIDRIÁN TORTI, J. L. (1998): "El Paleolítico Inferior y Medio en Andalucía", Arte, Arqueología e Historia 5: 19-38. Córdoba.

CORTÉS SÁNCHEZ, M. y SIMÓN VALLEJO, Mª D. (1998): "Cueva Bajondillo (Torremolinos, Málaga), implicaciones para el conocimiento de la dinámica cultural del Pleistoceno Superior en Andalucía", en SANCHIDRIÁN TORTI, J. L. y SIMÓN VALLEJO, Mª . D.: Las culturas del Pleistoceno Superior en Andalucía, I Simposio de Prehistoria Cueva de Nerja, Homenaje al profesor Dr. D. Francisco Jordá Cerdá, Patronato de la Cueva de Nerja: 35-62. Málaga.

CORTÉS SÁNCHEZ, M. (2000): "Bajondillo Cave (Torremolinos, Málaga, Andalucía) and the Middle-Upper Palaeolithic Transition in Southern Spain”, en STRINGER, C. B.; BARTON, R. N.E. y FINLAYSON, J. C. (Eds.): Neanderthals on the Edge: 123-132. Oxford.

CORTÉS SÁNCHEZ, M. y SIMÓN VALLEJO, Ma D. (2000): "Bahía de Málaga: algunos aspectos fisiográficos y su incidencia sobre los yacimientos arqueológicos pleistocenos en medio kárstico de su ámbito de influencia", I Congreso Andaluz de Espeleología: 217-224. Sevilla.

CORTÉS SÁNCHEZ, M. y SIMÓN VALLEJO, Mª D. (2001): “Cave Bajondillo (Torremolinos, Málaga, Andalucía). News of the transition between the Middle and Upper Palaeolithic in the South of Iberian Peninsula", en Les premiers hommes modernes de la Péninsule Ibérique, Actes du Colloque de la Comisión VIII de l'UISPP: 103-115. Lisboa. 
DURÁN, J.J.; GRÜN, R. y SORIA, J.M. (1988): “Edad de las formaciones travertínicas del flanco meridional de la Sierra de Mijas (provincia de Málaga, Cordilleras Béticas)", Geogaceta 5: 61 y ss. Madrid.

MARQUÉS MERELO, I. y FERRER PALMA, J. E. (1991): "Hallazgo de industria solutreogravetiense en el Bajondillo (Torremolinos, Málaga)", Baetica 13: 139-146. Málaga.

MUÑIZ PÉREZ, M. y PANIAGUA PÉREZ, J. P. (1996): "I Simposio de Prehistoria de Nerja", Espacio Tiempo y Forma. Prehistoria y Arqueología 9: 331-337. Madrid.

PANIAGUA PÉREZ, J.P. (1998): "El final de los complejos musterienses y los comienzos del paleolítico Superior en el Sur de la Península Ibérica", Spal 7: 45-67. Sevilla.

SANCHIDRIÁN TORTI, J.L. (1990): El Arte Paleolítico en Andalucía. Tesis Doctoral inédita, Universidad de Málaga.

SANCHIDRIÁN TORTI, J. L.; SIMÓN VALLEJO, Ma. D.; CORTÉS SÁNCHEZ, M. y MUÑOZ VIVAS, V. E. (1996): "La dinámica de los grupos predadores en la Prehistoria andaluza. Ensayo de síntesis", en CORTÉS SÁNCHEZ, M.; MUÑOZ VIVAS, V. E.; SANCHIDRIÁN TORTI, J. L. y SIMÓN VALLEJO, M. D., El Paleolítico en Andalucía: 13-87. Córdoba.

SANCHIDRIÁN TORTI, J.L. (1998): "Crónica", en SANCHIDRIÁN TORTI, J. L. y SIMÓN VALLEJO, $\mathrm{M}^{\mathrm{a}}$. D.: Las culturas del Pleistoceno Superior en Andalucía, I Simposio de Prehistoria Cueva de Nerja, Homenaje al profesor Dr. D. Francisco Jordá Cerdá, Patronato de la Cueva de Nerja: 35-62. Málaga. (2000): "Panorama actual del arte paleolítico en Andalucía", en OLIVEIRA JORGE, V. (Coord.): Paleolítico da Península Ibérica. Actas do $3^{\circ}$ Congresso de Arqueología Peninsular: 541-554.

ZILHAO, J. (2000): "The Ebro frontier: a model for the Late extincion of Iberian Neanderthals", en STRINGER, C. B.; BARTON, R. N.E. y FINLAYSON, J.C. (Eds.): Neanderthals on the Edge: 111-121. Oxford. 


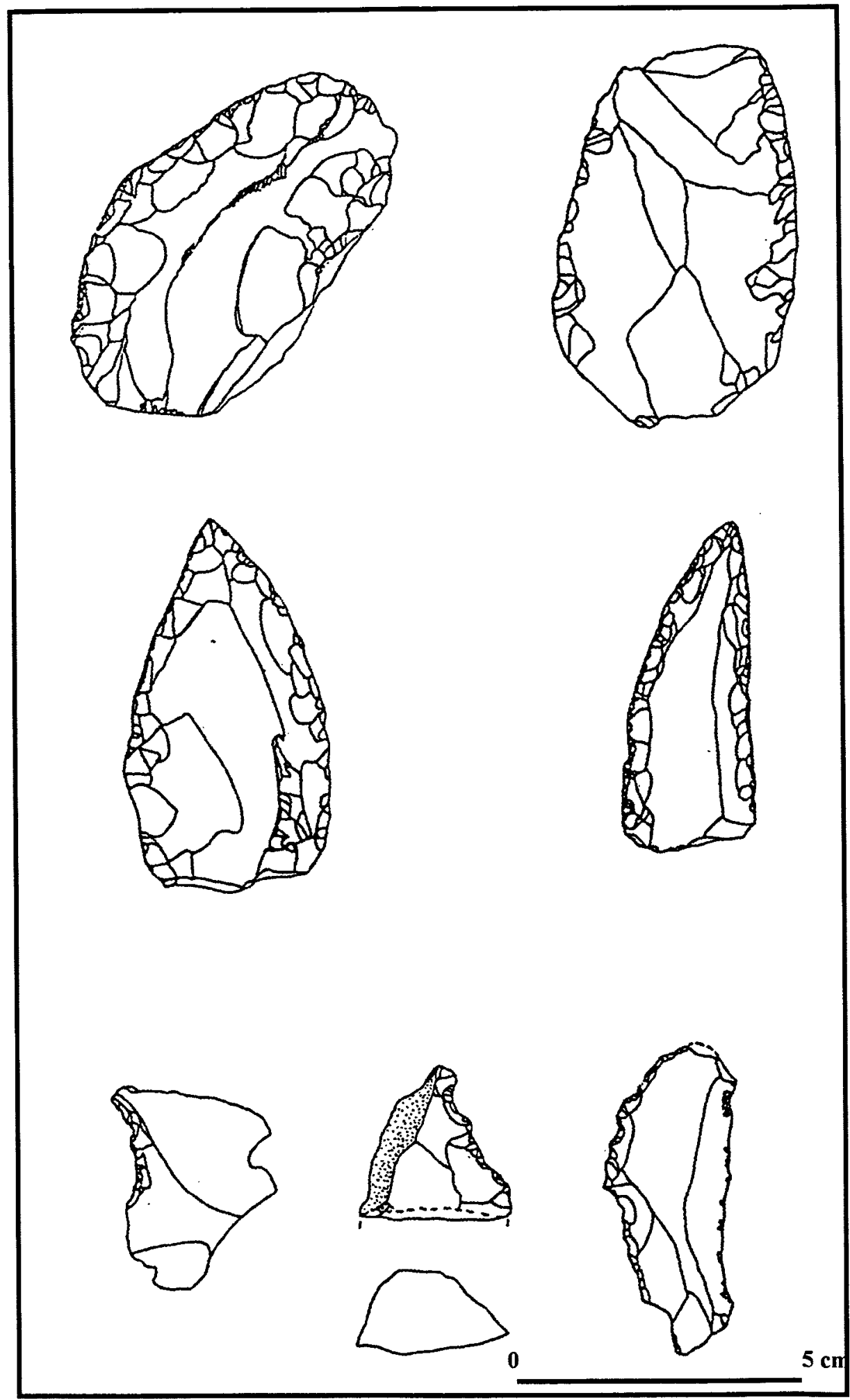

Figura 1: Materiales de los niveles musterienses. 


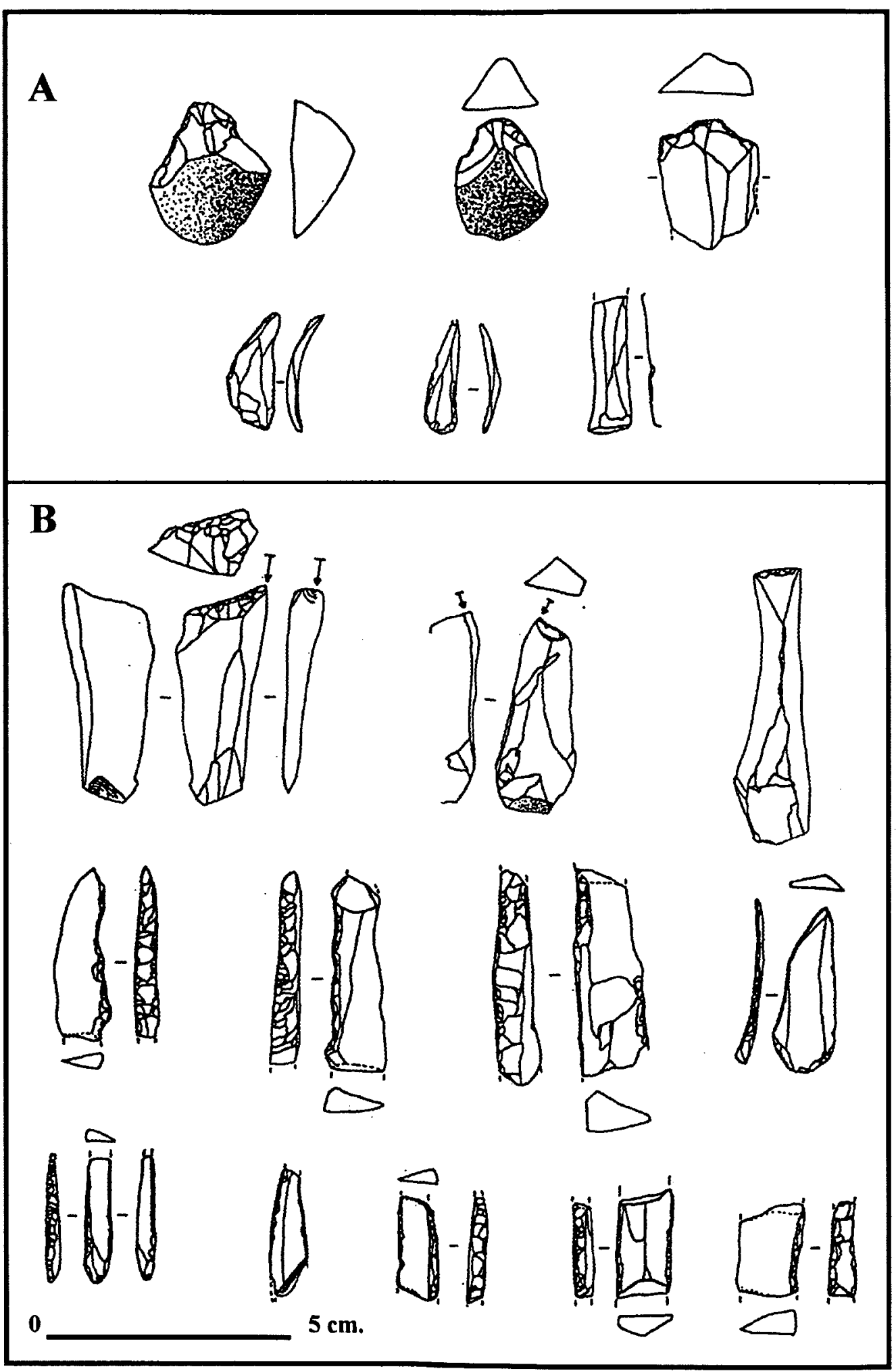

Figura 2-A: Materiales del nivel auriñaciense; B: materiales del nivel gravetiense. 


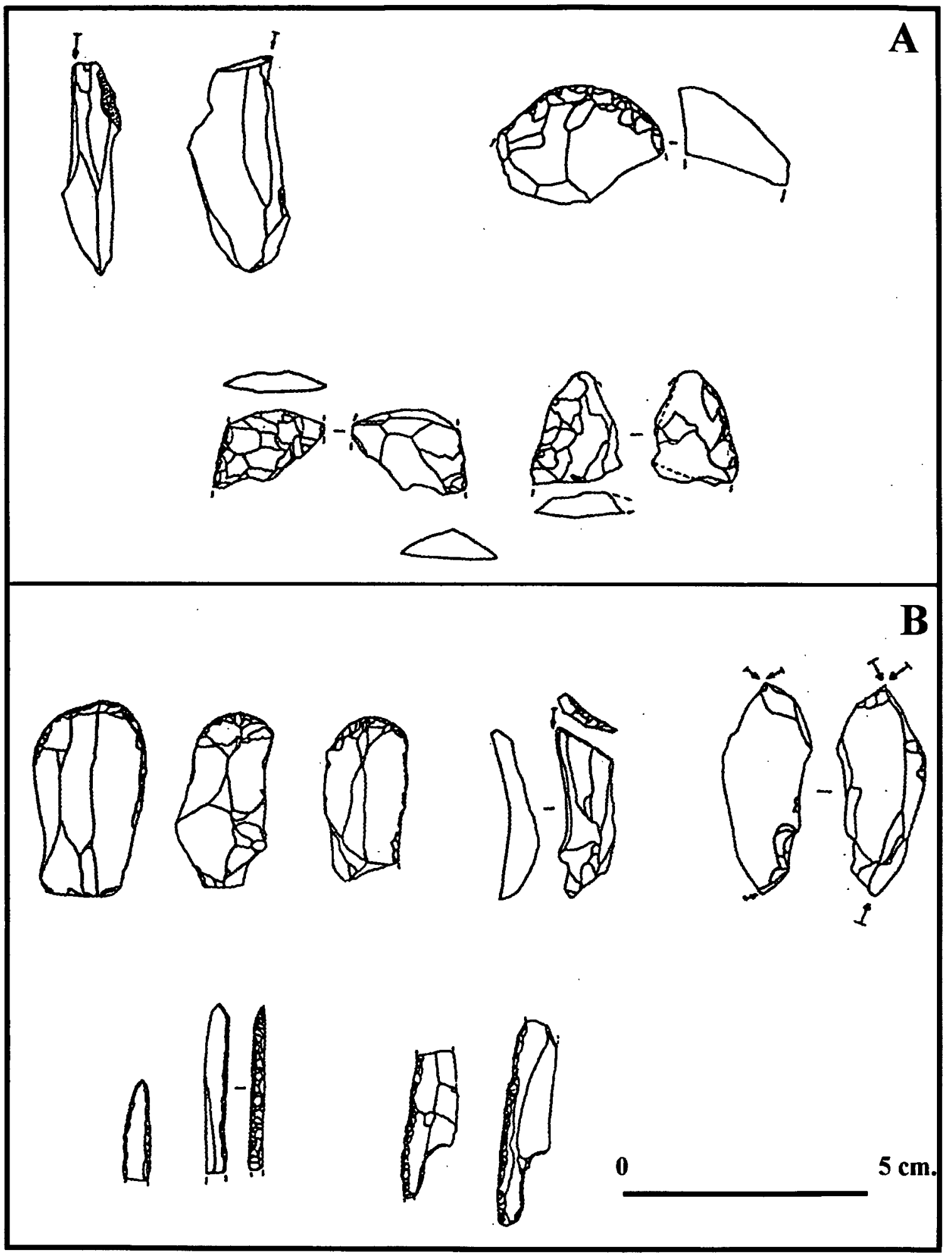

Figura 3-A: Materiales del solutrense pleno; B: materiales del nivel solutreogravetiense. 